\title{
On the Conditional Equivalence of Two Starting Methods for the Second Algorithm of Remez
}

\author{
By R. E. Huddleston
}

\begin{abstract}
In computing best min-max rational approximations by the second algorithm of Remez (which is an iterative procedure), one must provide a starting approximation. A method proposed by Ralston and one by Werner are shown to be equivalent under reasonable conditions.
\end{abstract}

1. Introduction. Ralston [3, p. 286] and Werner, Stoer, and Bommas [4] (hereinafter referred to as Werner) have suggested methods for obtaining a starting approximation for the Remez algorithm for rational Chebyshev approximation which work well in all but the hardest cases. (Both Ralston and Werner have also proposed methods for the remaining cases but those results will not be discussed here.) Werner's method is much easier to implement than Ralston's and, as we shall show here, produces the same starting approximation under very reasonable conditions.

2. Werner's Method. Let $f \in C[a, b]$ be the function which is to be approximated by a rational function of degree $m$ in the numerator and $n$ in the denominator. Denote the starting approximation by

$$
R_{m, n}^{(0)}(x)=\frac{P_{m}^{(0)}(x)}{Q_{n}^{(0)}(x)}=\frac{\sum_{i=0}^{m} a_{j} T_{i}(x)}{\sum_{j=0}^{n} b_{j} T_{i}(x)},
$$

where $T_{r}(x)=\cos (r \operatorname{arcos} x)$ is the Chebyshev polynomial of degree $r$.

Without loss of generality we assume that $[a, b]=[-1,1]$ and that $b_{0}=1$. To determine the $m+n+1$ coefficients $a_{i}, b_{i}$ Werner suggests the rational interpolation

$$
R_{m, n}^{(0)}\left(Z_{i}\right)=f\left(Z_{i}\right), \quad i=0,1, \cdots, m+n+1,
$$

where the $Z_{i}$ are the zeroes of the Chebyshev polynomial of degree $m+n+1$ which are given by

$$
Z_{i}=\cos ((2 i+1) \pi / 2(m+n+1))
$$

3. Ralston's Method. Obtaining the starting approximation (2.1) by Ralston's

Received April 2, 1973.

AMS (MOS) subject classifications (1970). Primary 41A20; Secondary 26A81, 65D20.

Key words and phrases. Approximation by rational functions, min-max rational approximations, Remez algorithm, starting procedures for min-max rational approximations.

Copyright @ 1974, American Mathematical Society 
method is a two part procedure. First one must determine the coefficients $C_{i}$ in the Chebyshev expansion

$$
f(x) \cong \frac{1}{2} C_{0}+\sum_{i=1}^{m+2 n} C_{i} T_{i}(x)
$$

where

$$
C_{i}=\frac{2}{\pi} \int_{-1}^{1} \frac{f(x) T_{i}(x)}{\left(1-x^{2}\right)^{1 / 2}} d x, \quad j=0,1, \cdots, m+2 n .
$$

Then one must solve the linear system

$$
\begin{aligned}
& a_{0}=\frac{1}{2} \sum_{i=0}^{n} b_{i} C_{i}, \\
& a_{r}=\frac{1}{2} \sum_{i=0}^{n} b_{i}\left(C_{|r-i|}+C_{r+i}\right), \quad r=1, \cdots, m+n,
\end{aligned}
$$

where $a_{r}=0$ for $r>m$.

If we express $f$ as

$$
f(x)=\frac{1}{2} C_{0}+\sum_{i=1}^{\infty} C_{i} T_{i}(x)
$$

then we may write

$$
f(x)-R_{m, n}^{(0)}(x)=N(x) / \sum_{i=0}^{n} b_{i} T_{i}(x)
$$

where

$$
N(x)=\left[\frac{1}{2} C_{0}+\sum_{i=1}^{\infty} C_{j} T_{j}(x)\right]\left[\sum_{i=0}^{n} b_{i} T_{i}(x)\right]-\sum_{i=0}^{m} a_{j} T_{j}(x) .
$$

Requiring that the coefficients of $T_{i}(x)$ in $N(x)$ vanish for $j=0,1, \cdots, m+n$ leads to the linear system (3.3).

4. Conditional Equivalence. In Ralston's procedure one must evaluate the integrals (3.2). The solution of (3.3) requires $m+2 n$ of the Chebyshev coefficients $C_{i}$. This is as opposed to the evaluation of $f$ at only $m+n+1$ points in Werner's method. Thus, on the surface these two linear systems, (2.2) and (3.3), do not seem equivalent. In order to establish a connection between them, we shall use two known identities concerning Chebyshev polynomials (see [2, p. 215]):

$$
\begin{aligned}
\sum_{i=0}^{m+n} T_{r}\left(Z_{i}\right) T_{i}\left(Z_{i}\right) & =0, & & 0 \leqq r \neq j \leqq m+n, \\
& =(m+n+1) / 2, & & 0<r=j \leqq m+n, \\
& =m+n+1, & & 0=r=j,
\end{aligned}
$$

where the $Z_{i}$ are given by (2.3), and

$$
T_{r+i}(x)+T_{|r-i|}(x)=2 T_{r}(x) T_{j}(x) .
$$

Let us rewrite the linear system (2.2) for Werner's approximation as 


$$
\sum_{i=0}^{m} a_{i} T_{i}\left(Z_{i}\right)=\sum_{i=0}^{n} b_{i} f\left(Z_{i}\right) T_{i}\left(Z_{i}\right) .
$$

Multiplying both sides by $T_{r}\left(Z_{i}\right)$ and summing over $i$ we have

$$
\sum_{i=0}^{m}\left(a_{i} \sum_{i=0}^{m+n} T_{i}\left(Z_{i}\right) T_{r}\left(Z_{i}\right)\right)=\sum_{i=0}^{n}\left(b_{i} \sum_{i=0}^{m+n} f\left(Z_{i}\right) T_{i}\left(Z_{i}\right) T_{r}\left(Z_{i}\right)\right) .
$$

Using (4.1) we have

$$
\sum_{i=0}^{m}\left(a_{i} \delta_{i r}\left[\begin{array}{cc}
(m+n+1) / 2, & j=r>0 \\
m+n+1, & j=r=0
\end{array}\right]\right)=\sum_{i=0}^{n}\left(b_{i} \sum_{i=0}^{m+n} f\left(Z_{i}\right) T_{i}\left(Z_{i}\right) T_{r}\left(Z_{i}\right)\right)
$$

where $\delta_{i r}$ is the Kronecker delta function. For $r=0$ this yields

$$
a_{0}=\sum_{i=0}^{n} b_{i}\left(\frac{1}{m+n+1} \sum_{i=0}^{m+n} f\left(Z_{i}\right) T_{i}\left(Z_{i}\right) T_{0}\left(Z_{i}\right)\right) .
$$

For $1 \leqq r \leqq m$, we have

$$
a_{r}=\sum_{i=0}^{n} b_{i}\left(\frac{2}{m+n+1} \sum_{i=0}^{m+n} f\left(Z_{i}\right) T_{i}\left(Z_{i}\right) T_{r}\left(Z_{i}\right)\right),
$$

and, for $r>m$,

$$
0=\sum_{i=0}^{n} b_{i}\left(\frac{2}{m+n+1} \sum_{i=0}^{m+n} f\left(Z_{i}\right) T_{i}\left(Z_{i}\right) T_{r}\left(Z_{i}\right)\right) .
$$

If we now employ (4.2) we have

$$
a_{0}=\frac{1}{2} \sum_{i=0}^{n} b_{i}\left(\frac{2}{m+n+1} \sum_{i=0}^{m+n} f\left(Z_{i}\right) T_{i}\left(Z_{i}\right)\right)
$$

and

$$
a_{r}=\frac{1}{2} \sum_{i=0}^{n} b_{i}\left(\left(\frac{2}{m+n+1} \sum_{i=0}^{m+n} f\left(Z_{i}\right) T_{r+j}\left(Z_{i}\right)\right)\right.
$$

$$
\left.+\left(\frac{2}{m+n+1} \sum_{i=0}^{m+n} f\left(Z_{i}\right) T_{|r-j|}\left(Z_{i}\right)\right)\right)
$$

for $1 \leqq r \leqq m+n$ where $a_{r}=0$ for $r>m$. We note that (4.3) looks a bit more like (3.3) now. In fact, they would be equal if the $C_{k}$, as defined by (3.2), were equal to $(2 /(m+n+1)) \sum_{i=0}^{m+n} f\left(Z_{i}\right) T_{k}\left(Z_{i}\right)$. Using the Gauss-Chebyshev quadrature rule (see [3, p. 99]), we have

$$
C_{k}=\frac{2}{\pi} \int_{-1}^{1} \frac{f(x) T_{k}(x)}{\left(1-x^{2}\right)^{1 / 2}} d x=\frac{2}{m+n+1} \sum_{i=0}^{m+n} f\left(Z_{i}\right) T_{k}\left(Z_{i}\right)+E,
$$

where

$$
E=\frac{1}{2^{2(m+n)}(2 m+2 n) !} \frac{d^{(2 m+2 n+2)}}{d x^{(2 m+2 n+2)}}\left(f(\eta) T_{k}(\eta)\right) .
$$

Thus, under the condition that the Gauss-Chebyshev quadrature rule with $m+$ $n+1$ points is used, the two methods of obtaining a starting approximation are 
equivalent. In practice one would find it rare to be able to exactly evaluate integrals such as (3.2) - thus some kind of quadrature rule must be used. Keeping in mind that we are trying to derive a starting approximation for an iterative procedure, the error given by $E$ seems sufficiently small.

A FORTRAN implementation of the ALGOL procedure by Werner, Stoer, and Bommas [4] and a document [1] describing the implementation is available from the author.

Numerical Applications Division 8441

Sandia Laboratories

Livermore, California 94550

1. R. E. Huddleston, REHRAT-A Program for Best Min-Max Rational Approximation, Report \#(SCL-DR-72-370), Sandia Laboratories, Livermore, California, 1972.

2. E. IsAacson \& H. B. KelleR, Analysis of Numerical Methods, Wiley, New York, 1966. MR 34 \#924.

3. A. RAlston, A First Course in Numerical Analysis, McGraw-Hill, New York, 1965. MR $32 \# 8479$.

4. H. K. E. Werner, J. Stoer \& W. Bommas, "Rational Chebyshev approximation," Numer. Math., v. 10, 1967, pp. 289-306. 